\title{
OPEN Investigating BB0405 as a novel Borrelia afzelii vaccination candidate in Lyme borreliosis
}

\author{
M. J. Klouwens ${ }^{1,2,3 凶}$, J. J. Trentelman ${ }^{1}$, J. I. Ersoz ${ }^{1}$, F. Nieves Marques Porto ${ }^{1}$, R. Sima ${ }^{4}$, \\ O. Hajdusek ${ }^{4}$, M. Thakur ${ }^{5}$, U. Pal ${ }^{5}$ \& J. W. Hovius ${ }^{1,2,3}$
}

BB0405 is a surface exposed Borrelia burgdorferi protein and its vaccination protected mice against $B$. burgdorferi infection. As BB0405 is highly conserved across different $B$. burgdorferi sensu lato species, we investigated whether vaccination with recombinant BB0405 or through intradermal bb0405 DNA tattoo vaccination could provide protection against different Borrelia species, specifically against Borrelia afzelii, the predominant $B$. burgdorferi sensu lato genospecies causing Lyme borreliosis across Eurasia. We immunized $\mathrm{C} 3 \mathrm{H} / \mathrm{HeN}$ mice with recombinant $\mathrm{BB} 0405$ or with a codon-optimized $b 60405$ DNA vaccine using the pVAC plasmid and immunized corresponding control groups mice with only adjuvant or empty vectors. We subsequently subjected these immunized mice to a tick challenge with B. afzelii CB43-infected Ixodes ricinus nymphs. Upon vaccination, recombinant BB0405 induced a high total IgG response, but b60405 DNA vaccination did not elicit antibody responses. Both vaccine formulations did not provide protection against Borrelia afzelii strain CB43 after tick challenge. In an attempt to understand the lack of protection of the recombinant vaccine, we determined expression of BB0405 and showed that $B$. afzelii CB43 spirochetes significantly and drastically downregulate the expression of $\mathrm{BB} 0405$ protein at $37^{\circ} \mathrm{C}$ compared to $33^{\circ} \mathrm{C}$, where as in $\mathrm{B}$. burgdorferi $\mathrm{B} 31$ spirochetes expression levels remain unaltered. Vaccination with recombinant BB0405 was previously shown to protect against $B$. burgdorferi sensu stricto. Here we show that vaccination with either recombinant BB0405 (or non-immunogenic bb0405 DNA), despite being highly conserved among $B$. burgdorferi sl genospecies, does not provide cross-protection against $B$. afzelii, mostly likely due to downregulation of this protein in B. afzelii in the mammalian host.

Lyme borreliosis is the most common vector-borne disease in the Northern hemisphere and is caused by spirochetes belonging to the Borrelia burgdorferi sensu lato (sl) group. They are transmitted by Ixodes ticks and although humans get infected by $B$. burgdorferi sl, they are accidental hosts and do not play a role in the spirochete's enzootic life cycle ${ }^{1}$. Because $B$. burgdorferi sl are extracellular pathogens, the outer membrane of the spirochete, containing multiple surface exposed lipoproteins, is continuously exposed to the immune system of the host ${ }^{2,3}$. Many studies have focused on identifying new B. burgdorferi outer surface proteins (Osps), because these are key targets for the host's humoral immune response and thus may be potential new vaccinogens $s^{4}$. Indeed, multiple protective Osps have been identified and OspA formed the basis of the only anti-Lyme vaccine that was publically available ${ }^{5}$. There is however a wide genetic diversity among B. burgdorferi sl genospecies and the spirochetes change surface proteins throughout their life cycle, which makes it challenging to identify protective antigens ${ }^{1}$.

Originally, Brooks et al. identified several surface-exposed B. burgdorferi sensu stricto (ss) outer membrane proteins to which specific anti-B. burgdorferi antibodies were shown to be bactericidal ${ }^{6}$. Among these proteins was BB0405, an outer membrane protein unique for B. burgdorferi sl species. With 78 to $90 \%$ identity between BB0405 orthologues, its sequence is highly conserved among B. burgdorferi ss, B afzelii and B garinii, the three major genospecies causing Lyme borreliosis ${ }^{7}$. Although initially thought not to be immunogenic during natural

\footnotetext{
${ }^{1}$ Department of Internal Medicine, Center for Experimental and Molecular Medicine, Academic Medical Center, University of Amsterdam, Meibergdreef 9, 1105 AZ Amsterdam, The Netherlands. 2Division of Infectious Diseases, Department of Internal Medicine, Academic Medical Center, Amsterdam, The Netherlands. ${ }^{3}$ Amsterdam Multidisciplinary Lyme Borreliosis Center, Academic Medical Center, Amsterdam, The Netherlands. ${ }^{4}$ Biology Centre, Institute of Parasitology, Czech Academy of Sciences, Ceske Budejovice, Czech Republic. ${ }^{5}$ Department of Veterinary Medicine, University of Maryland, College Park and Virginia- Maryland Regional College of Veterinary Medicine, College Park, MD, USA.『email:m.j.klouwens@amsterdamumc.nl
} 


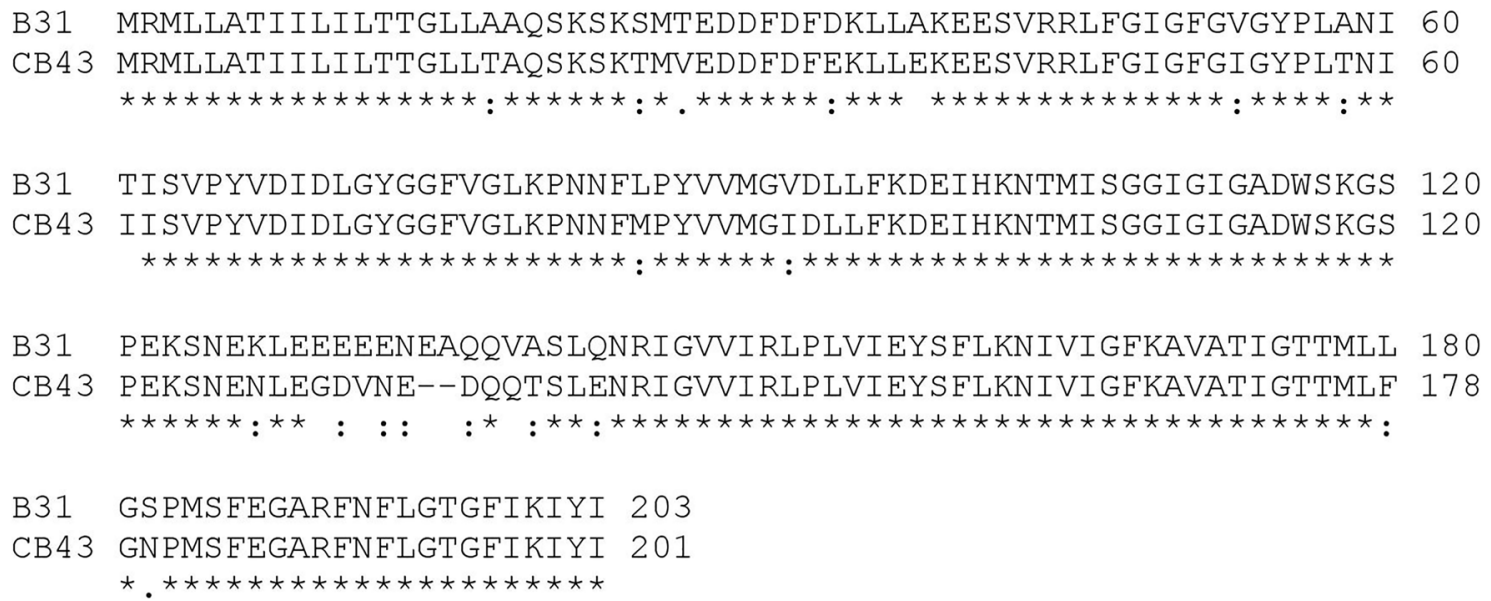

Figure 1. Alignment between the BB0405 protein sequence between Borrelia burgdorferi $\mathrm{B} 31$ and Borrelia afzelii PKO. Borrelia burgdorferi B31 corresponds to NCBI Reference Sequence: NP_212539. Borrelia afzelii PKO is identical to CB43 (GenBank: CP002933.1 translated in ExPASy translate tool). Identity between sequences is $88.2 \%$. Additionally there are 17 similar positions bringing the similarity to $96.5 \%$. Alignment was performed with Clustal Omega. ${ }^{*}=$ identical, $:=$ similar.

infection ${ }^{8}$ it was shown by Brooks et al. that BB0405 is immunogenic and actively expressed during nonhuman primate infection by $B$. burgdorferi by detecting the protein with sera from infected baboons ${ }^{6,8}$. Multiple studies show that BB0405 is necessary for establishing infection in mice, since bb0405-deletion mutants are unable to be transmitted from ticks and establish infection in mammalian hosts ${ }^{7,8}$. Of importance, vaccination with recombinant BB0 405 also protected mice from $B$. burgdorferi infection by B. burgdorferi-infected ticks ${ }^{7,8}$. Thus, $b b 0405$ is a highly conserved antigen with the potential to form the basis for a vaccine protecting against multiple B. burgdorferi sl genospecies.

Most research on new Lyme vaccines focuses on recombinant proteins, but DNA vaccination constitutes an alternative vaccination platform ${ }^{9}$. For instance, a previous study by Wagemakers et al. has shown that DNA vaccination by tattoo with $B$. afzelii strain $\mathrm{PKo}$ Outer surface protein $\mathrm{C}(\mathrm{OspC})$ was fully protective against $B$. afzelii challenge in mice and induced favorable humoral immune responses compared to recombinant protein vaccination $^{10}$. In line with this, we were able to show protection against B. burgdorferi strain $\mathrm{N} 40$ in a similar set-up, in which OspC from B. burgdorferi strain N40 was used both as recombinant as well as DNA vaccine (Klouwens et al. manuscript in preparation).

In the current study we aimed to investigate the role of BB0405 in providing protection across $B$. burgdorferi sl genospecies. To this end, mice were immunized with B. burgdorferi B31-derived recombinant BB0405 or bb0405 DNA vaccine and subsequently challenged with B. afzelii CB43-infected ticks, after which immunogenicity and host protection of the two different vaccination approaches were determined using established methods.

\section{Results}

Immunogenicity of $\mathbf{b} b \mathbf{0 4 0 5}$ antigens. As described previously, BB0405 is a highly conserved B. burgdorferi sl surface protein and alignment of the protein BB0405 of B. burgdorferi B31 and B. afzelii CB43 showed an identity of $88 \%$ and similarity of $96 \%$ at the amino acid sequence level (Fig. 1). To determine whether antibodies against BB0405 would protect across different $B$. burgdorferi sl genospecies, we performed a vaccination study in mice. Recombinant BB0405 and a DNA vaccine for bb0405, both generated from B. burgdorferi B31, were constructed as well as an empty DNA vaccine, functioning as the negative control. From our previous published and unpublished studies it is known that an empty DNA vaccine, i.e. a pVAX vector without inserted target sequences, does not affect B. burgdorferi sl infection ( ${ }^{10}$ and Klouwens et al. manuscript in preparation). Two weeks after the 3 rd vaccination, mice were challenged with B. afzelii CB43-infected Ixodes ricinus nymphs. To assess immunogenicity, BB0405 specific total IgG levels were measured by ELISA before and after vaccinations (Fig. 2). As expected, the BB0405 specific total IgG levels were very high in the mice that had received the recombinant $\mathrm{BB} 0405$ vaccine. In contrast, this was not the case in the mice that had received the bb0405 DNA vaccine. In fact, the antibody titers in this group were comparable to the control mice that had been vaccinated with the empty DNA vaccine.

Host-protection via BB0405 immunization. Next, we assessed whether both vaccines were able to provide protection against $B$. afzelii infection by performing qPCR and culture of several tissues, obtained by sacrificing the mice 21 days after challenge with $B$. afzelii strain CB43-infected ticks. B. afzelii loads in ear, skin (tick feeding site), heart, bladder and ankle were quantified and normalized to mouse $\beta$-actin. In addition, bladder and skin tissues from the feeding site of the ticks were cultured and checked weekly for the growth of spirochetes. Despite the high antibody titers, results of qPCR and culture indicate that vaccination with BB0405, either recombinant or as DNA vaccine, did not protect against heterologous Borrelia challenge in mice (Table 1). 
BB0405 antibody response $t=42$ pre-challenge

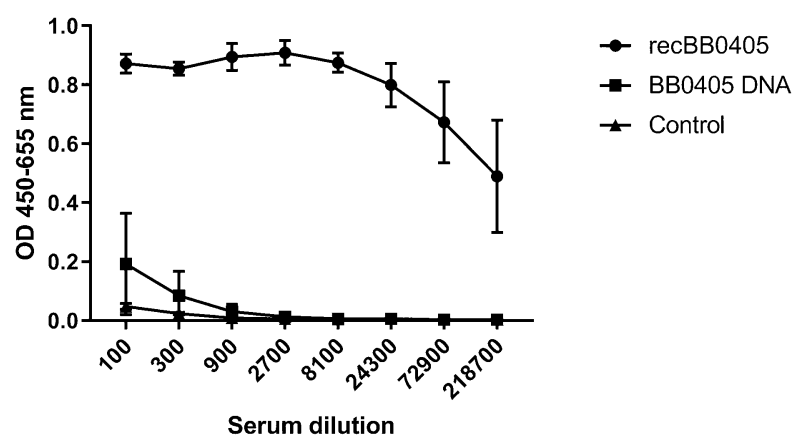

Figure 2. Enzyme-linked immunosorbent assay (ELISA) showing high-titer antibodies induced in recombinant BB0405 immunized mice. BB0405 specific total IgG responses were measured in mice sera of three vaccination groups with 8 mice each. IgG responses are presented as optical density (OD) 450-655 nm for multiple sera dilutions. The first group was immunized at three time points (0, 14 and 28 days) with recBB0405. The second groups received DNA vaccinations at the same time points with DNA BB0405 and the third group with DNA Empty (negative control). Plates were coated with recombinant BB0405 and incubated with mouse sera, which was collected from mice at time point 42 days, just before tick challenge and after three vaccinations. Sera was diluted in steps of 300 until 1:218700.

\begin{tabular}{|l|l|l|l|l|l|l|l|l|}
\hline \multirow{2}{*}{ Vaccin } & \multicolumn{4}{|l|}{} & \multicolumn{2}{c|}{$\begin{array}{l}\text { Culture } \\
\text { qPCR positivity }\end{array}$} & \multirow{2}{*}{ posity } \\
\cline { 2 - 8 } & Skin & Ear & Bladder & Heart & Ankle & Skin & Bladder & Cumulative positivity \\
\hline Rec BB0405 & $4 / 8$ & $6 / 8$ & $2 / 8$ & $4 / 8$ & $6 / 8$ & $4 / 8$ & $4 / 8$ & $8 / 8$ \\
\hline DNA BB0405 & $3 / 8$ & $4 / 8$ & $1 / 8$ & $2 / 8$ & $4 / 8$ & $2 / 8$ & $2 / 8$ & $5 / 8$ \\
\hline Control & $5 / 8$ & $4 / 8$ & $4 / 8$ & $3 / 8$ & $3 / 8$ & $3 / 8$ & $2 / 8$ & $8 / 8$ \\
\hline
\end{tabular}

Table 1. Overview of number of Borrelia positive mice per group in qPCR tissues and culture. Each experimental group consisted of 8 mice. qPCR was performed on 5 different tissues: skin (tick bite site on the back), ear, bladder, heart and ankle. Both skin and half of the bladder were cultured in MKP medium and checked for growth of Borrelia for 8 weeks. Statistical significance of cumulative data of the experimental groups compared to the DNA Empty was calculated with the Fisher's exact test. Comparison between all separate groups was not statistically significant.

Indeed, there were no significant differences between the separate groups in the different tissues, nor between experimental groups at a cumulative level.

BB0405 surface expression B. burgdorferi sensu stricto and B. afzelii. To study the location of the protein and also the potential accessibility of the protein to antibodies we performed a proteinase $\mathrm{K}$ assay with viable B. burgdorferi ss and B. afzelii spirochetes followed by Western Blot analysis. We show that BB0405 is partly surface exposed as the protein is partly digested upon proteinase $\mathrm{K}$ treatment for both 30 and $60 \mathrm{~min}$ both in B. burgdorferi ss as well as in B. afzelii. Flagellin B, a periplasmic control protein, was, as expected, not affected by proteinase K treatment (Fig. 3).

Expression of BB0405 or homologous proteins in $B$. burgdorferi sensu stricto and $B$. afzelii. Finally, in an attempt to understand why, in contrast to previous observations for B. burgdorferi $\mathrm{ss}^{7,8}$, BB0405 did not protect against B. afzelii, we next precisely compared the expression levels of BB0405 between these two different $B$. burgdorferi sl genospecies under different experimental conditions. We did not find differences in expression of bb0405 at the RNA level between B. burgdorferi ss and B. afzelii grown at $33{ }^{\circ} \mathrm{C}$ or $37^{\circ} \mathrm{C}$ (data not shown). However, we also assessed BB0405 expression at the protein level. To this end, equal amounts of whole lysates of spirochetes, cultivated in vitro at different temperatures, were run on a Western blot and incubated with BB0405 specific antibodies. As can be appreciated from Fig. 4A, B. afzelii CB43 produces considerably lower amounts of BB0405 at $37^{\circ} \mathrm{C}$, the condition similar to the conditions in the mammalian host. We also quantified the protein bands on the Western Blots (Image J software) and measured the relative density of the bands compared to a corresponding Flagellin B loading control (Fig. 4B). Indeed, the relative density of BB0405 in B. afzelli CB43 cultured at $37^{\circ} \mathrm{C}$ was significantly lower compared to BB0405 expression at $33^{\circ} \mathrm{C}$ and to $B$. burgdorferi $\mathrm{B} 31$ at $37^{\circ} \mathrm{C}$, offering a possible explanation for our findings. 


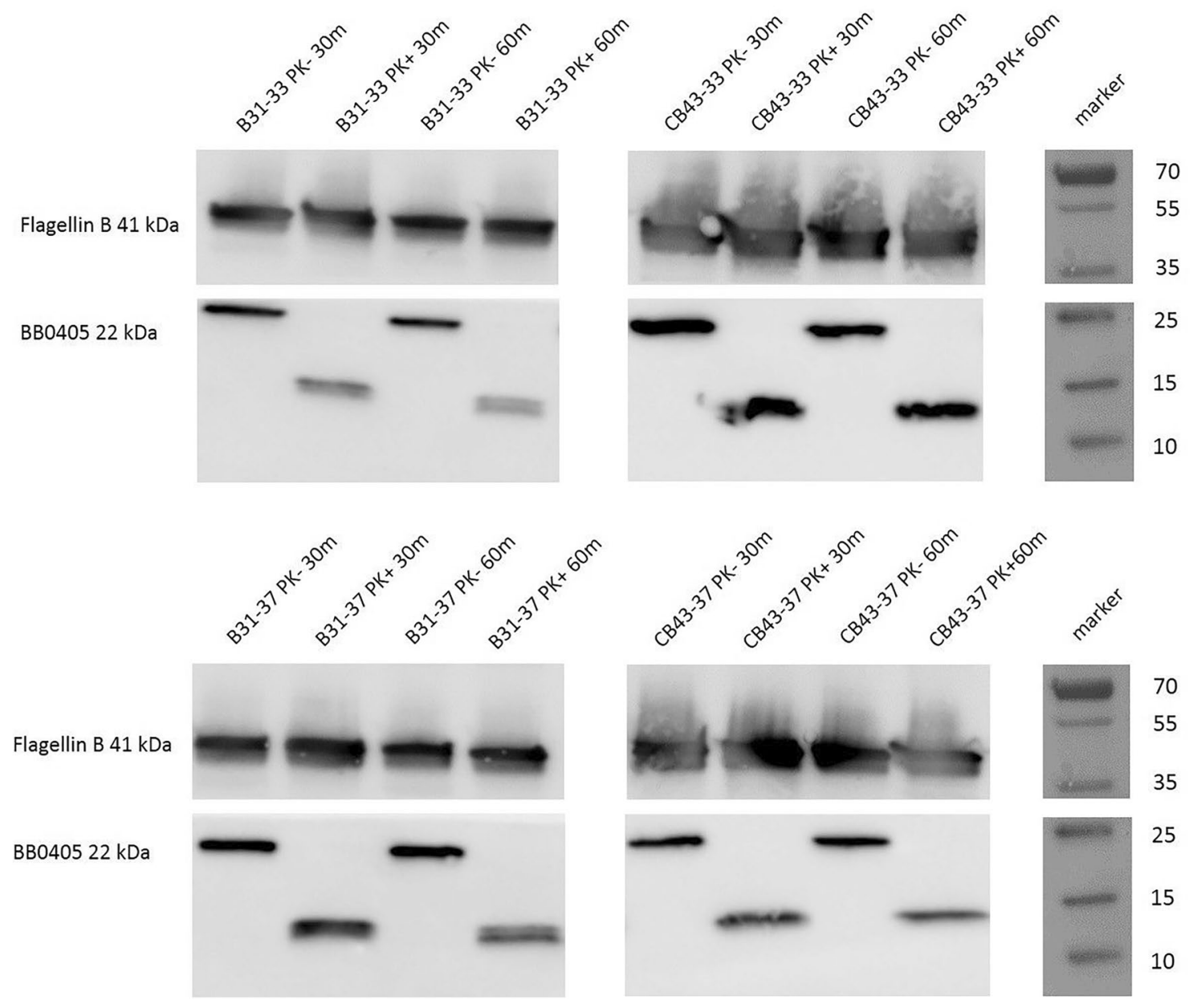

Figure 3. BB0405 is expressed on the surface of B. burgdorferi sensu stricto and B. afzelii. Viable spirochetes were incubated with $(+)$ or without $(-)$ proteinase $\mathrm{K}$ for $30 \mathrm{~min}$ and $60 \mathrm{~min}$ and processed for immunoblot analyses with antibodies against BB0405. Flagellin B antibodies served as a subsurface control.

\section{Discussion}

An ideal Lyme vaccine candidate would need to provide protection against the most dominant B. burgdorferi sl genospecies causing Lyme borreliosis. The main B. burgdorferi sl genospecies causing Lyme borreliosis in NorthAmerica is B. burgdorferiss, whereas in Europe this is B. afzelii. To explore the potential of BB0405, in the current study we investigated whether vaccination with BB0405-a surface-exposed B. burgdorferi ss protein that is well-conserved among different $B$. burgdorferi sl genospecies including $B$. afzelii-protected against heterologous challenge with $B$. afzelii through tick-bite in an experimental mouse model. Moreover, two different methods of vaccination with BB0405 were used, recombinant protein vaccination and DNA vaccination by tattoo. Finally, to provide further insights into our findings we assessed the differential BB0405 expression of B. burgdorferi ss and $B$. afzelii under varying conditions.

An adequate humoral response is essential in clearing Borrelia spirochetes and specific immunoglobulins play a key role in affording protective host immunity ${ }^{11-13}$. Since BB0405 is an outer surface protein, it is surface exposed and therefore likely to be antibody-accessible. Surprisingly, Kung et al. described the interesting phenomenon that no BB0405 specific antibodies are generated during natural infection in mice; however Brooks et al. showed that recombinant BB0405 is immunogenic in rats and antibodies raised against BB0405 could kill $B$. burgdorferi ss in vitro in the presence of complement ${ }^{6}$. In addition, in mice immunized with recombinant BB0405 a strong and long-lasting antibody response was induced that provided protective immunity against tick-transmitted infection with $B$. burgdorfer $i^{8}$. In the current study we also show a robust humoral immune response in mice vaccinated with recombinant BB0 405 compared to control mice, corroborating the observation that recombinant BB0405 is immunogenic. In contrast, we did not observe antibody responses against BB0405 in the mice vaccinated with the $b b 0405$ DNA vaccine. Interestingly, the same DNA vaccination approach has been successful in the past for other Borrelia outer surface proteins ${ }^{10}$. In our study this could indicate that the mice cells were not able to transcribe, translate or translocate $b b 0405$ and that the utility of DNA vaccination against B. burgdorferi sl is highly dependent on the target. However, as murine infection with $B$. burgdorferi ss, either by syringe or ticks, does not result in BB0405-specific antibody responses, it could also be that $b b 0405$ needs the adjuvants used in recombinant protein vaccinations and is not immunogenic by itself ${ }^{8}$. 


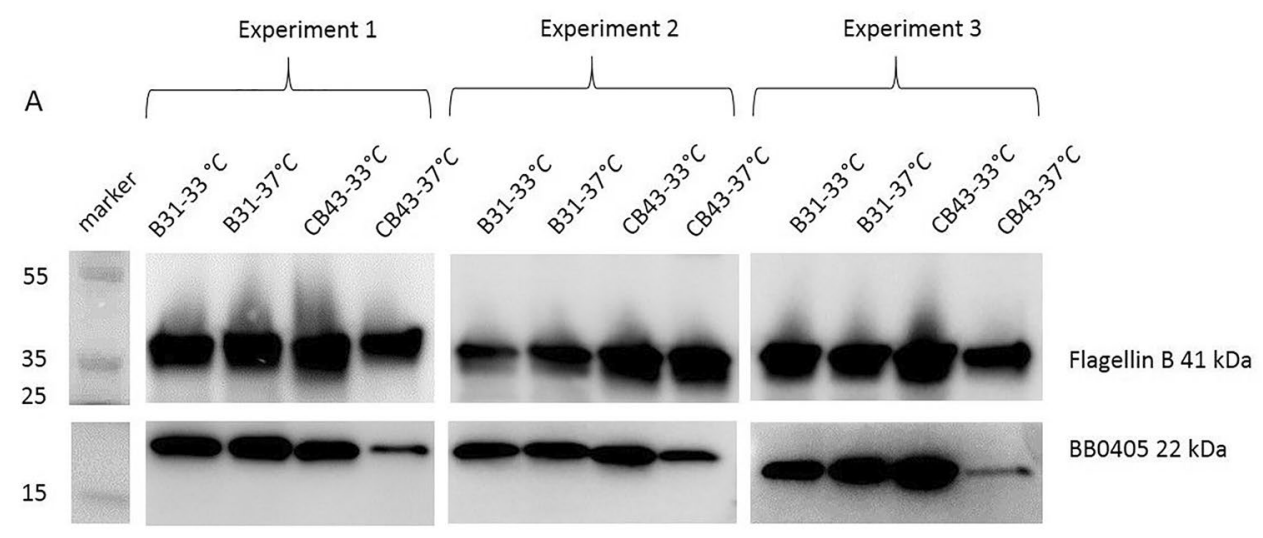

B

BB0405 relative protein expression in different Borrelia strains, grown at different temperatures

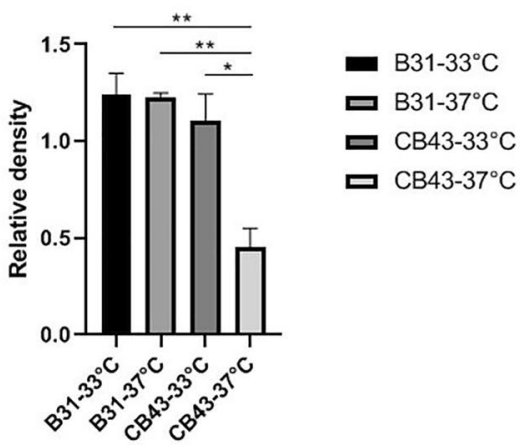

Figure 4. (A) Western blot showing expression of BB0405 in different B. burgdorferi sl strains (Borrelia burgdorferi strain B31 and Borrelia afzelii strain CB43) grown at different temperatures. A temperature of $33^{\circ} \mathrm{C}$, resembling (feeding) tick conditions, and $37^{\circ} \mathrm{C}$, resembling conditions in the mammalian host, was used to culture the spirochetes. Lysates of the in vitro cultured spirochetes were obtained, as described in the materials and method section, and subjected to Western blot. Three independent experiments were performed. The first experiment is displayed on the left, the second experiment in the middle and the third experiment is displayed on the right. The far left lane is the protein weight marker. Blots were loaded with $2.5 \mathrm{ug} /$ well whole lysates of spirochetes and were cut in half just between the bands of Flagellin B (41 kDa) and BB0405 (22 kDa) to enable separate incubations. The blots displayed at the top are incubated with anti-flagellin rabbit IgG 1:1000 (as a loading control) and subsequently with secondary antibody anti-rabbit IgG-HRP 1:1000. Blots displayed at the bottom are incubated with pooled serum from mice vaccinated with recombinant BB0405 1:500 and subsequently with secondary antibody anti-mouse IgG-HRP 1:2000, respectively. Blot images were cropped (Image acquisition tools Microsoft Powerpoint). Imaging was performed using ImageQuant LAS 4000 and quantification using Image J (Wayne Rasband, National Institutes of Health, USA, Java 1.8.0_77(32-bit), http:// imagej.nih.gov/ij). Full length blots are presented in Supplementary Fig. S1-S6. (B) Protein expression of BB0405 as determined by Western blot in panel A was quantified and normalized against the relative density of loading control Flagellin B. Relative density was determined using ImageJ software (National Institute of Health). A quantitative comparison between samples on the same blot and within the same experiment were made. Statistical differences between groups were calculated using an unpaired parametric t-test. Error bars represent mean \pm s.e.m. n.s.: $P>0.05$.

In the study described here, we have challenged the BB0405 (B. burgdorferi B31 derived)-immunized mice with $B$. afzelii infected ticks. We did not observe any protection of BB0405, neither as recombinant vaccine nor as DNA tattoo. Based on our observations, BB0405 does not seem to be a suitable vaccine candidate for the European situation, as it does not provide cross-protection between B. burgdorferi sl species-in this case $B$. burgdorferi ss and $B$. afzelii-despite high antibody titers after vaccination with recombinant protein. Although we have performed a heterologous challenge, the in silico analysis showed high sequence homology between these proteins. Indeed in Figs. 3 and 4 we show that the raised antibodies recognize and bind to BB0405 expressed by $B$. afzelii. It seems therefore unlikely that antigen recognition can explain the observed lack of vaccine efficacy. Future studies could assess whether using different species-specific BB0405 homologues from different B. burgdorferi sl genospecies could protect against heterologous challenge. 
As stated above, antigen specificity of the generated antibodies is unlikely to explain the observed lack of efficacy. Therefore we firstly wanted to determine whether BB0405 was indeed surface expressed in B. afzelii strain CB43, as has been shown B. burgdorferi ss strain B31 ${ }^{8}$. We have shown that BB0405 is indeed partly expressed at the cell surface by performing a Western Blot analysis after treating viable $B$ afzelii $B 43$ spirochetes with proteinase $\mathrm{K}$. We used $B$. burgdorferi $\mathrm{B} 31$ as a control. The reason that there is only partial surface expression in both $B$. burgdorferi sl strains can be explained by BB0405 being a transmembrane protein and thus partly intracellular or membrane bound, explaining the lower protein products on the Western Blots of Fig. 3. Secondly, there are over 20 different species within the B. burgdorferi sl complex and these are capable of molecular adaptations in order to ensure efficient transmission by their vector and to survive in different environments of their hosts ${ }^{14-18}$. Temperature is a key environmental factor known to affect $B$. burgdorferi sl gene expression. More specifically, it has been described by Ojaimi et al. that BB0405 is upregulated by temperature suggesting upregulation in conditions that mimic the situation in the mammalian host ${ }^{17}$. We therefore wondered whether a difference in protein expression of BB0405 between American and European B. burgdorferi sl genospecies could explain the lack of protection in our vaccination experiment. At the protein level we observed a distinct and significantly lower expression of BB0405 in B. afzelii CB43 spirochetes grown at $37^{\circ} \mathrm{C}$, as compared to the expression in the same spirochetes grown at $33^{\circ} \mathrm{C}$. More importantly, expression of BB0405 in B. afzelii CB43 spirochetes grown at $37^{\circ} \mathrm{C}$ was also significantly lower compared to B. burgdorferi $\mathrm{B} 31$ spirochetes grown at the same temperatures. This difference was not apparent at the RNA level. However, it is well-known that RNA expression levels do not necessarily correspond to the protein expression levels ${ }^{19}$. Thus, the BB0405 protein seems to be evidently less expressed by B. afzelii CB43 in conditions reflecting the mammalian host and this might explain the observed lack of protection against $B$. afzelii strain CB43, despite the presence of specific antibodies upon vaccination with recombinant BB0405. An alternative explanation for the lack of protection could be an indirect effect of failure of antibody recognition. B. burgdorferi sl is able to alter surface protein in vivo via differential gene expression and via VlsE recombination which might impact spirochete recognition by host-generated antibodies ${ }^{20-23}$. There are multiple examples of surface-exposed Borrelial antigens that are masked by neighboring (abundant) proteins $s^{15,24}$. Although BB0405 is conserved across B. burgdorferi sl genospecies, it could be possible that expression of other surface proteins is different in B. burgdorferi ss and B. afzelii, which may account for different surface topology and outcome of antibody-mediated protections.

In conclusion, being a surface-exposed, immunogenic and well-conserved B. burgdorferi sl protein, BB0405 was shown to be an interesting vaccine candidate to protect against Lyme borreliosis caused by B. burgdorferi ss. However, we here show that vaccination with B. burgdorferi ss-derived BB0405 does not protect against heterologous challenge with B. afzelii through tick-bite, and our data suggest that this could be due to lower expression of the BB0405 homologue at the protein level in B. afzelii in the mammalian host. Nevertheless, experiences with the OspA vaccine have shown that a vaccine does not have to protect against all B. burgdorferi sl genospecies to be commercially viable. Future experiments should investigate whether multivalent BB0 405 vaccines are able to protect against multiple $B$. burgdorferi sl genospecies.

\section{Methods}

Ethics statement. All experiments were reviewed and approved by the Animal Research Ethics Committee of the Academic Medical Center, Amsterdam, The Netherlands (protocol 208AK-1 and 271AA-1). Experiments have been conducted according to European and national guidelines http://eur-lex.europa.eu/legal-content/EN/ TXT/?uri=celex:32010L0063 ) and in compliance with the ARRIVE guidelines (http://www.nc3rs.org.uk/page. asp?id=1357).

Recombinant BB0405 protein. The bb0405 gene was cloned without the $\mathrm{N}$-terminal leader sequence into pET28a (Invitrogen), produced in E. coli, and purified using Ni-NTA resin as detailed elsewhere ${ }^{8}$. Sequence alignment between BB0405 from different strains was performed using Clustal Omega ${ }^{25}$.

Generation of the BB0405 DNA vaccine. The pVAXhTPA-BB0405 DNA vaccine was designed as described before in Wagemakers et al. ${ }^{10}$. In the BB0405 gene sequence of B. burgdorferi B31 (NCBI Reference Sequence: NC_001318.1) the 18aa signal sequence (predicted by SignalP 4.0 web-based software, CBS, Lyngby, Denmark) was replaced with the human tissue plasminogen activator (hTPA) signal sequence (genbank AAA61213.1 $)^{26}$. The resulting sequence was codon-optimized to mouse tRNA usage with Java Codon Adaptation tool (Braunschweig, Germany) ${ }^{27}$. At the $5^{\prime}$ end a BamH1 and a Kozak sequence were added, and at the 3' end a sequence encoding a double stop codon and a Xhol were added. The insert was synthesized (BaseClear, Leiden, The Netherlands) and ligated into a BamH1/Xho1 restricted empty pVAX vector (Invitrogen, Carlsbad, CA, USA). The plasmid was amplified using a Nucleobond Xtra EF kit (Macherey-Nagel, Düren, Germany) and resuspended in DNase free water.

Vaccination experiments. Six to eight weeks old female $\mathrm{C} 3 \mathrm{H} / \mathrm{HeN}$ mice were purchased from Charles River and the experiment was designed with 3 groups of eight mice each. The vaccination experiment was carried out as described in a previous publication of our group ${ }^{10}$. The first group was vaccinated with a recombinant BB0405 vaccine (recBB0405), the second group with a BB0405 DNA vaccine, and the third group consisted of an empty vector DNA vaccination group as a negative control. Mice were vaccinated at $t=0, t=14$ and $t=28$ days and sera were collected at each time point for use in ELISA experiments. For the recombinant BB0405 vaccine $10 \mu \mathrm{g}$ protein was emulsified with complete Freund's adjuvant at $\mathrm{t}=0$ and $5 \mu \mathrm{g}$ in incomplete Freund's adjuvant at $t=14$ and $t=28$ days (Fig. 5). All vaccinations were administered subcutaneously. For the pVAX-hTPABB0405 DNA vaccine and the negative control hair was removed from the mice abdomens using hair removal 


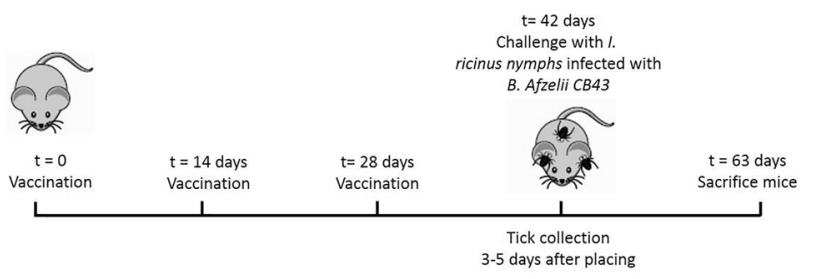

Figure 5. Schematic design of the vaccination study. The study consisted of 3 experimental groups of 8 mice each. The first group was vaccinated with recombinant BB0405, the second group was received a tattooed DNA vaccination with BB0405 and the third group received an empty vector DNA vaccination as a control. Mice were vaccinated at $\mathrm{t}=0, \mathrm{t}=14$ and $\mathrm{t}=28$ days. They were challenged with infected ticks 2 weeks after the last booster vaccination and sacrificed at day 63.

cream (Veet). Using a Cheyenne Hawk tattoo machine carrying a Cheyenne 13-magnum tattoo needle (both MT.DERM, Berlin, Germany) $20 \mu \mathrm{g}$ of the DNA vaccines was tattooed $0.5-1 \mathrm{~mm}$ into the abdominal skin of the mice for $45 \mathrm{~s}$ at $100 \mathrm{~Hz}$ under isofluorane anesthesia. Two weeks after the third vaccination, at $\mathrm{t}=42$, all mice were challenged with 5 Ixodes ricinus nymphs infected with Borrelia afzelii strain CB43. These B. afzeliiinfected I. ricinus nymphs were placed in a containing capsule and fed on the immunized and control animals until repletion. Additional sera were collected at $\mathrm{t}=42$ (pre-challenge, 2 weeks after the third immunization) and at $\mathrm{t}=63$ days ( 3 weeks post-challenge) mice were sacrificed and ear, skin, ankle, heart, bladder and tissue was collected for analysis. Mice were considered to be infected when there was at least one positive tissue sample in either qPCR or culture.

ELISA. To measure IgG directed against BB0405, ELISAs were performed according to previous described protocol $^{10}$. High-binding 96-well ELISA plates (Greiner Bio-one, Kremsmünster, Austria) were coated overnight at $4{ }^{\circ} \mathrm{C}$ with $1 \mu \mathrm{g} / \mathrm{ml}$ recBB0405, washed with PBS-Tween (phosphate-buffered saline- $0.05 \%$ Tween) and incubated with blocking buffer (1\% BSA in PBS) for $2 \mathrm{~h}$ at room temperature. Mouse sera (collected at day 42 before tick challenge) were diluted in blocking buffer, added to the wells and incubated for $1 \mathrm{~h}$ at room temperature. Plates were washed and incubated for $1 \mathrm{~h}$ with horseradish peroxidase (HRP)-linked anti-mouse IgG (Cell Signaling, Beverly, MA, USA) diluted 1:1000 in blocking buffer. The plates were washed again and developed using TMB substrate $(50 \mu \mathrm{l} \mathrm{TMB}$ chromogene in $5 \mathrm{ml}$ TMB substrate buffer $(8.2 \mathrm{~g} \mathrm{NaAc}$ and 21 gr citric acid monohydrate dissolved in $1 \mathrm{~L} \mathrm{H}_{2} \mathrm{O}+10 \mu \mathrm{l} 3 \% \mathrm{H}_{2} \mathrm{O}_{2}$ ) and optical density was measured in a Biotek (Winooski, VT, USA) ELISA plate reader at $450-655 \mathrm{~nm}$.

Borrelia afzelii detection and quantification. Murine bladder and skin samples were cultured in modified Kelly Pettenkofer (MKP) medium with rifampicin, $50 \mu \mathrm{g} / \mathrm{ml}$ and phosphomycin, $100 \mu \mathrm{g} / \mathrm{ml}$ ) at $33^{\circ} \mathrm{C}$. The cultures were checked weekly (for a total of 8 weeks) for the presence of motile spirochetes with dark field microscopy as described before ${ }^{28}$. For all samples DNA was extracted using Qiagen Blood and Tissue kit (Qiagen, Venlo, The Netherlands). Quantitative (q)PCR was used to quantify B. afzelii DNA in mouse tissues and was performed according to previously described protocol ${ }^{10}$. OspA primers were used for quantification; forward 5'-AAAAATATTTATTGGGAATAGGTCT-3' and reverse 5'-CACCAGGCAAATCTACTGAA-3', mouse Beta-actin forward $5^{\prime}$-AGCGGGAAATCGTGCGTG-3' and reverse primer $5^{\prime}$-CAGGGTACATGGTGGTGC C-3' were used for normalization. The qPCRs were performed on the LightCycler480 (Roche, Nutley, NJ, USA) using SYBR green dye (Roche) using the following PCR protocol: $95^{\circ} \mathrm{C} 6 \mathrm{~min}$, and 60 cycles of $95^{\circ} \mathrm{C} 10 \mathrm{~s}, 60^{\circ} \mathrm{C}$ $20 \mathrm{~s}$ and $72^{\circ} \mathrm{C} 20 \mathrm{~s}$. Reactions were performed in triplicate. Results were analyzed using LinRegPCR software (Amsterdam, The Netherlands) ${ }^{29}$. Negative and positive controls were included in each qPCR run. A positive Borrelia load was determined by at least one melting curve exactly matching the positive control (skin tissue known to be infected with $B$. afzelii). In case of a negative value for OspA in a sample, the OspA value was replaced by the value of the OspA detection limit. The detection limit is defined as the highest dilution of the control sample in which OspA is still detectable + 3xSD.

RNA and cDNA synthesis. B. burgdorferi $\mathrm{B} 31$ and $B$. afzelii $\mathrm{CB} 43$ were cultured in $\mathrm{MKP}$ medium at $33^{\circ} \mathrm{C}$ or $37^{\circ} \mathrm{C}$ to $1 \times 10 \mathrm{e} 6$ spirochetes/ $\mathrm{ml}$ as assessed by a Petroff-Hausser counting chamber and dark-field microscopy. The cultures were centrifuged for $10 \mathrm{~min}$ at $10.000 \mathrm{rpm}$ and the pellets were dissolved in $750 \mu \mathrm{RNA}$ Later (Qiagen) stored at $-80^{\circ} \mathrm{C}$ until further use. All samples were thawed simultaneously and centrifuged for $5 \mathrm{~min}$ at $12.000 \mathrm{rpm}$. The pellets were then subsequently used for isolation of RNA using the Nucleospin RNA isolation kit (Macherey-Nagel), according to the manufacturer's instructions. Subsequently, RNA samples were digested by DNase for a second time using the Qiagen RNase-Free DNase Set (\#79254) and then cleaned up using the RNeasy MinElute Cleanup Kit (\#74104, Qiagen). A total of $10 \mu \mathrm{l}$ of each RNA sample was then used to generate cDNA and heated for $5 \mathrm{~min}$ at $85^{\circ} \mathrm{C}$ and then cooled to $23^{\circ} \mathrm{C}$. To every sample, $10 \mu \mathrm{l}$ of RT mix was added, consisting of $4 \mu \mathrm{l}$ of M-MLV reverse transcriptase buffer (Promega), $0.5 \mu \mathrm{l}$ of M-MLV reverse transcriptase enzyme $200 \mathrm{U} / \mathrm{ml}$ (Promega), $2 \mu \mathrm{l}$ dNTP mix (Invitrogen), $1 \mu \mathrm{l}$ of random hexamers (), $0.5 \mu \mathrm{l}$ DTT (Invitrogen), $0.5 \mu \mathrm{R}$ RaseOut (Invitrogen), as well as $1.5 \mu \mathrm{l}$ of RNase free $\mathrm{H} 2 \mathrm{O}$. The PCR protocol for all samples was $23^{\circ} \mathrm{C}$ $10 \mathrm{~min}, 42^{\circ} \mathrm{C} 60 \mathrm{~min}$ and $95^{\circ} \mathrm{C} 3 \mathrm{~min}$ (2720 Thermal Cycler, Applied Biosystems). To check the purity of the RNA, a PCR was performed using RNA and cDNA (100× diluted) of corresponding samples as a template with 
universal $f l a B$ primers ( $f l a B$ forward 5'-GCTTCTGATGATGCTGCTG-3' and $f l a B$ reverse 5'-CGTCTGTAA GTTGCTCTATTTC-3') and PCR Phusion High Fidelity Mastermix (\#M0531S, NEB). As expected, there was no amplification in the samples were RNA was used as template, indicating there was no DNA contamination. Real-time quantitative polymerase chain reaction (qRT-PCR) was used to quantify the amount of BB0405 RNA in the two different $B$. burgdorferi sl strains, grown at different temperatures. The qRT-PCR was performed with BB0405 specific primers for B. afzelii CB43 (forward 5'- GTCTGTGCGCCGTTTATTTG-3' and reverse 5'-GGG TTTAAGTCCAACGAATCC-3') and for B. burgdorferi B31 (forward 5'-GGCATAGGTTTTGGAGTTGG-3' and reverse 5'-CCATCACAACATAGGGCAAG-3') and with Flagellin B universal primers as housekeeping genes (forward 5'-GCTTCTGATGATGCTGCTG-3' and reverse 5'-CGTCTGTAAGTTGCTCTATTTC-3'). The cDNA from the two different $B$. burgdorferi sl strains grown under different conditions was diluted 100 times and $1 \mu \mathrm{l}$ was used as template per reaction and qPCRs were performed using the LightCycler480 (Roche, Nutley, NJ, USA) and SYBR green dye (Roche) in triplicate. The PCR protocol was $95^{\circ} \mathrm{C} 6 \mathrm{~min}$, and 60 cycles of $95^{\circ} \mathrm{C}$ $10 \mathrm{~s}, 60^{\circ} \mathrm{C} 20 \mathrm{~s}$ and $72^{\circ} \mathrm{C} 20 \mathrm{~s}$. Results were analyzed using LinRegPCR software (Amsterdam, The Netherlands).

Borrelia lysates and BB0405 western blots. B. burgdorferi B31 and B. afzelii $\mathrm{CB} 43$ were cultured in MKP medium at $33{ }^{\circ} \mathrm{C}$ or $37^{\circ} \mathrm{C}$ until log phase was reached, after which the cultures were centrifuged and the spirochete pellets were washed three times in PBS for $10 \mathrm{~min}$ at $10.000 \mathrm{~g}$. Ultimately, the pellets were dissolved in $250 \mu \mathrm{l}$ of PBS and were sonicated on ice (6 times $15 \mathrm{~s}$ on, $30 \mathrm{~s}$ off with $20 \%$ amplitude using Vibra-Cell High Intensity ultrasonic processor). Protein concentrations were measured using the Pierce BCA protein assay (\#23225 from Thermo Scientific). Subsequently, lysates (2.5 $\mu$ g protein per sample) were mixed in a 5:1 ratio with $5 \times$ SDS sample reducing buffer (1.5\% SDS, $10 \%$ glycerol, $62.5 \mathrm{mM}$ Tris- $\mathrm{HCl}$ (Ph 6.8), 2\%-mercapto-ethanol and $0.0025 \%$ bromophenol blue) and subjected to sodium dodecyl sulfate-polyacrylamide gel electrophoresis (SDSPAGE) using a precast SDS 4-20\% polyacrylamide gel (Bio-Rad Mini-PROTEAN TGX) with PageRuler Plus Prestained Protein Ladder (Thermo Scientific). Samples were then transferred to a 99\% ethanol-activated PVDF membrane. After blotting, the membrane was cut in half just between the bands of Flagellin $\mathrm{B}(41 \mathrm{kDa})$ and BB0405 $(22 \mathrm{kDa})$ to enable separate incubations. The upper half of the membrane, containing Flagellin B, was incubated with a 1:1.000 dilution of anti-flagellin rabbit IgG (Anti-Flagellin RABBIT Antibody, \#200-401-C14S, Rockland) as a loading control. The lower half of the membrane, containing BB0405, was incubated with a 1:500 dilution of murine sera derived from mice immunized with recombinant BB0405 (B. burgdorferi B31). Immunoblots were subsequently labeled with HRP-conjugated secondary antibodies (anti-rabbit IgG-HRP 1:1000 and anti-mouse IgG-HRP 1:2000 respectively, by Cell Signaling) and developed using Pierce ECL Western Blotting Substrate (\#32106 Thermo scientific). Imaging was performed using ImageQuant LAS 4000 and quantification using Image J (Wayne Rasband, National Institutes of Health, USA, Java 1.8.0_77(32-bit), http://imagej.nih.gov/ ij).

Proteinase K assay. Proteinase K assays were carried out as described elsewhere $\mathrm{F}^{30-32}$. Spirochetes in midphase growth were washed twice with $\mathrm{PBS}+5 \mathrm{mM} \mathrm{MgCl}_{2}$ and were pelleted. Pellets were resuspended gently and split in equal volumes. They were incubated in PBS $5 \mathrm{mM} \mathrm{MgCl}_{2}$ either in the absence or presence of proteinase $\mathrm{K}$ at a concentration of $200 \mu \mathrm{g} / \mathrm{ml}$ for either 30 or $60 \mathrm{~min}$ at room temperature. Phenylmethylsulfonyl floride (PMSF)(Sigma) was added $(1.5 \mathrm{mM})$ to stop proteinase $\mathrm{K}$ activity. Spirochetes were again washed twice in PBS $+5 \mathrm{mM} \mathrm{MgCl}_{2}+1 \mathrm{mM}$ PMSF and pelleted. The pellets were resuspended in an appropriate volume of SDS-PAGE loading buffer for further Western Blot analysis. Western Blots were carried out as described above.

Statistic methods. Differences between experimental groups between Borrelia loads in qPCR were statistically tested by two-sided nonparametric tests (Mann-Whitney, GraphPad Prism software version 5.0, San Diego, CA, USA). Differences in expression level between experimental groups on Western Blot were compared using an unpaired t test (Graphpad Prism software version 5.0, San Diego, CA, USA).

Received: 17 July 2020; Accepted: 1 February 2021

Published online: 26 February 2021

\section{References}

1. Radolf, J. D., Caimano, M. J., Stevenson, B. \& Hu, L. T. Of ticks, mice and men: Understanding the dual-host lifestyle of Lyme disease spirochaetes. Nat. Rev. Microbiol. 10, 87-99. https://doi.org/10.1038/nrmicro2714 (2012).

2. Hovius, J. W., van Dam, A. P. \& Fikrig, E. Tick-host-pathogen interactions in Lyme borreliosis. Trends Parasitol. 23, 434-438. https ://doi.org/10.1016/j.pt.2007.07.001 (2007).

3. Pulzova, L. \& Bhide, M. Outer surface proteins of Borrelia: Peerless immune evasion tools. Curr. Protein Pept. Sci. 15, 75-88. https ://doi.org/10.2174/1389203715666140221124213 (2014).

4. Kenedy, M. R., Lenhart, T. R. \& Akins, D. R. The role of Borrelia burgdorferi outer surface proteins. FEMS Immunol. Med. Microbiol. 66, 1-19. https://doi.org/10.1111/j.1574-695X.2012.00980.x (2012).

5. Steere, A. C. et al. Vaccination against Lyme disease with recombinant Borrelia burgdorferi outer-surface lipoprotein A with adjuvant. Lyme Disease Vaccine Study Group. N. Engl. J. Med. 339, 209-215. https://doi.org/10.1056/nejm199807233390401 (1998).

6. Brooks, C. S., Vuppala, S. R., Jett, A. M. \& Akins, D. R. Identification of Borrelia burgdorferi outer surface proteins. Infect. Immun. 74, 296-304. https://doi.org/10.1128/iai.74.1.296-304.2006 (2006).

7. Shrestha, B., Kenedy, M. R. \& Akins, D. R. Outer membrane proteins BB0405 and BB0406 are immunogenic, but only BB0405 is required for Borrelia burgdorferi infection. Infect. Immun. 85, 66. https://doi.org/10.1128/iai.00803-16 (2017).

8. Kung, F. et al. A Borrelia burgdorferi surface-exposed transmembrane protein lacking detectable immune responses supports pathogen persistence and constitutes a vaccine target. J. Infect. Dis. 213, 1786-1795. https://doi.org/10.1093/infdis/jiw013 (2016). 
9. Bins, A. D. et al. A rapid and potent DNA vaccination strategy defined by in vivo monitoring of antigen expression. Nat. Med. 11, 899-904. https://doi.org/10.1038/nm1264 (2005).

10. Wagemakers, A. et al. Rapid outer-surface protein C DNA tattoo vaccination protects against Borrelia afzelii infection. Gene Ther. 21, 1051-1057. https://doi.org/10.1038/gt.2014.87 (2014).

11. Zhong, W. et al. Therapeutic passive vaccination against chronic Lyme disease in mice. Proc. Natl. Acad. Sci. USA 94, 12533-12538. https://doi.org/10.1073/pnas.94.23.12533 (1997).

12. Fikrig, E. et al. Sera from patients with chronic Lyme disease protect mice from Lyme borreliosis. J. Infect. Dis. 169, 568-574. https ://doi.org/10.1093/infdis/169.3.568 (1994).

13. Connolly, S. E. \& Benach, J. L. The versatile roles of antibodies in Borrelia infections. Nat. Rev. Microbiol. 3, 411-420. https://doi. org/10.1038/nrmicro1149 (2005).

14. Yang, X. et al. Analysis of Borrelia burgdorferi proteome and protein-protein interactions. Methods Mol. Biol. 1690, 259-277. https ://doi.org/10.1007/978-1-4939-7383-5_19(2018).

15. Yang, X. et al. Characterization of multiprotein complexes of the Borrelia burgdorferi outer membrane vesicles. J. Proteome Res. 10, 4556-4566. https://doi.org/10.1021/pr200395b (2011).

16. Phelan, J. P. et al. Genome-wide screen identifies novel genes required for Borrelia burgdorferi survival in its Ixodes tick vector. PLoS Pathog. 15, e1007644. https://doi.org/10.1371/journal.ppat.1007644 (2019).

17. Ojaimi, C. et al. Profiling of temperature-induced changes in Borrelia burgdorferi gene expression by using whole genome arrays. Infect. Immun. 71, 1689-1705. https://doi.org/10.1128/iai.71.4.1689-1705.2003 (2003).

18. Angel, T. E. et al. Proteome analysis of Borrelia burgdorferi response to environmental change. PLoS ONE 5, e13800. https://doi. org/10.1371/journal.pone.0013800 (2010).

19. Vogel, C. \& Marcotte, E. M. Insights into the regulation of protein abundance from proteomic and transcriptomic analyses. Nat. Rev. Genet. 13, 227-232. https://doi.org/10.1038/nrg3185 (2012).

20. Zhang, J. R. \& Norris, S. J. Genetic variation of the Borrelia burgdorferi gene vlsE involves cassette-specific, segmental gene conversion. Infect. Immun. 66, 3698-3704 (1998).

21. Bankhead, T. \& Chaconas, G. The role of VlsE antigenic variation in the Lyme disease spirochete: Persistence through a mechanism that differs from other pathogens. Mol. Microbiol. 65, 1547-1558. https://doi.org/10.1111/j.1365-2958.2007.05895.x (2007).

22. Coutte, L., Botkin, D. J., Gao, L. \& Norris, S. J. Detailed analysis of sequence changes occurring during vlsE antigenic variation in the mouse model of Borrelia burgdorferi infection. PLoS Pathog. 5, e1000293. https://doi.org/10.1371/journal.ppat.1000293 (2009).

23. Gilmore, R. D. Jr. et al. Temporal expression analysis of the Borrelia burgdorferi paralogous gene family 54 genes BBA64, BBA65, and BBA66 during persistent infection in mice. Infect. Immun. 75, 2753-2764. https://doi.org/10.1128/iai.00037-07 (2007).

24. Bunikis, J. \& Barbour, A. G. Access of antibody or trypsin to an integral outer membrane protein (P66) of Borrelia burgdorferi is hindered by Osp lipoproteins. Infect. Immun. 67, 2874-2883 (1999).

25. Consortium, T. U. UniProt: A worldwide hub of protein knowledge. Nucleic Acids Res. 47, D506-D515. https://doi.org/10.1093/ nar/gky1049 (2018).

26. Petersen, T. N., Brunak, S., von Heijne, G. \& Nielsen, H. SignalP 4.0: Discriminating signal peptides from transmembrane regions. Nat. Methods 8, 785-786. https://doi.org/10.1038/nmeth.1701 (2011).

27. Grote, A. et al. JCat: A novel tool to adapt codon usage of a target gene to its potential expression host. Nucleic Acids Res. 33, W526-531. https://doi.org/10.1093/nar/gki376 (2005).

28. Hovius, J. W. et al. Preferential protection of Borrelia burgdorferi sensu stricto by a Salp15 homologue in Ixodes ricinus saliva. J. Infect. Dis. 198, 1189-1197. https://doi.org/10.1086/591917 (2008).

29. Ruijter, J. M. et al. Amplification efficiency: Linking baseline and bias in the analysis of quantitative PCR data. Nucleic Acids Res. 37, e45. https://doi.org/10.1093/nar/gkp045 (2009).

30. Yang, X. et al. Characterization of unique regions of Borrelia burgdorferi surface-located membrane protein 1. Infect. Immun. 78, 4477-4487. https://doi.org/10.1128/iai.00501-10 (2010).

31. Coleman, A. S. et al. Borrelia burgdorferi complement regulator-acquiring surface protein 2 does not contribute to complement resistance or host infectivity. PLoS ONE 3, 3010e. https://doi.org/10.1371/journal.pone.0003010 (2008).

32. Mulay, V. et al. Borrelia burgdorferi BBA74, a periplasmic protein associated with the outer membrane, lacks porin-like properties. J. Bacteriol. 189, 2063-2068. https://doi.org/10.1128/jb.01239-06 (2007).

\section{Acknowledgements}

This project has received funding from the European Union's Seventh Programme for research, technological development and demonstration under grant agreement No. 602272 of which JWH was the scientific coordinator. RS and $\mathrm{OH}$ were supported from the grant Centre for Research of Pathogenicity and Virulence of Parasites (no. CZ.02.1.01/0.0/0.0/16_019/ 0000759), funded by the European Regional Development Fund (ERDF) and Ministry of Education, Youth, and Sport, Czech Republic (MEYS).

\section{Author contributions}

M.J.K. and J.J.T. designed the study. J.I.E., J.J.T. and M.J.K. performed the study procedures. F.N.M.P. assisted in the study procedures. R.S. and O.H. provided us with Ixodes ricinus nymphs infected with Borrelia afzelii CB43 and provided scientific input. U.P. and M.T. generated and provided the recombinant proteins and provided scientific input. J.W.H. supervised the study progress. All authors revised the draft manuscript and agreed to the final manuscript.

\section{Competing interests}

The authors declare no competing interests.

\section{Additional information}

Supplementary Information The online version contains supplementary material available at https://doi. org/10.1038/s41598-021-84130-y.

Correspondence and requests for materials should be addressed to M.J.K.

Reprints and permissions information is available at www.nature.com/reprints.

Publisher's note Springer Nature remains neutral with regard to jurisdictional claims in published maps and institutional affiliations. 
(c) (i) Open Access This article is licensed under a Creative Commons Attribution 4.0 International cc) License, which permits use, sharing, adaptation, distribution and reproduction in any medium or format, as long as you give appropriate credit to the original author(s) and the source, provide a link to the Creative Commons licence, and indicate if changes were made. The images or other third party material in this article are included in the article's Creative Commons licence, unless indicated otherwise in a credit line to the material. If material is not included in the article's Creative Commons licence and your intended use is not permitted by statutory regulation or exceeds the permitted use, you will need to obtain permission directly from the copyright holder. To view a copy of this licence, visit http://creativecommons.org/licenses/by/4.0/.

(C) The Author(s) 2021 